\title{
Usefulness of continuous blood glucose monitoring and control for patients undergoing liver transplantation
}

\author{
Tsuyoshi Okada', Shinji Kawahito², Naoji Mita², Munehide Matsuhisa ${ }^{3}$, \\ Hiroshi Kitahata ${ }^{4}$, Mitsuo Shimada ${ }^{5}$, and Shuzo Oshita ${ }^{2}$ \\ ${ }^{1}$ Department of Anesthesia, Takamatsu Red Cross Hospital, Kagawa, Japan, ${ }^{2}$ Department of Anes- \\ thesiology, Tokushima University Hospital, Tokushima, Japan, ${ }^{3}$ Diabetes Therapeutics and Research \\ Center, the University of Tokushima, Tokushima, Japan, ${ }^{4}$ Department of Dental Anesthesiology, \\ Tokushima University Hospital, Tokushima, Japan, ${ }^{5}$ Department of Digestive and Pediatric Surgery, \\ Tokushima University Hospital, Tokushima, Japan
}

\begin{abstract}
Background : The purpose of this study was to evaluate the usefulness of the closed-loop system (STG-22 ; Nikkiso, Tokyo, Japan), a type of artificial endocrine pancreas for the continuous monitoring and control of intraoperative blood glucose in patients undergoing liver transplantation. Methods : Sixteen patients undergoing livingdonor liver transplantation were enrolled in this study. Glucose levels were controlled with either a manual injection of insulin based on a commonly used sliding scale (manual insulin group, $n=8$ ) or a programmed infusion of insulin determined by the control algorithm of the artificial endocrine pancreas (programmed insulin group, $n=8$ ). The target glucose level range was set at $80-150 \mathrm{mg} / \mathrm{dl}$. Results : The mean and SD of blood glucose concentration during surgery (Glu-Ave and Glu-SD, respectively) for the programmed insulin group were lower than for the manual insulin group. The coefficient of variability (Glu-CV=Glu-SD $\times 100 /$ Glu-Ave) for the programmed insulin group was also lower than for the manual insulin group $(20.1 \pm 4.9 \%$ vs. $26.9 \pm 6.1 \%$; mean \pm SD). No hypoglycemia was detected in either group. Conclusion : The STG-22 closed-loop system is effective for maintaining strict blood glucose control during liver transplantation with minimal variability in blood glucose concentration. J. Med. Invest. 60 : 205-212, August, 2013
\end{abstract}

Keywords : continuous blood glucose monitoring, intensive insulin therapy, liver transplantation, glucose toxicity, artificial endocrine pancreas

\section{INTRODUCTION}

Liver transplantation is associated with marked hemodynamic, hematologic, and metabolic abnormalities $(1,2)$. Because the liver plays a key role in maintenance of blood glucose, one of the metabolic

Received for publication February 12, 2013 ; accepted February $25,2013$.

Address correspondence and reprint requests to Shinji Kawahito, $\mathrm{MD}, \mathrm{PhD}$, Department of Anesthesiology, Tokushima University Hospital, 3-18-15 Kuramoto, Tokushima 770-8503, Japan and Fax : +81-88-633-7182. disorders involves glucose metabolism, so that glucose regulation becomes a matter of concern during liver transplantation (3-6). Several recent clinical studies have demonstrated the efficacy of strict glucose control for reducing the mortality rate of postoperative or emergency patients $(7,8)$. This led to the notion that strict perioperative glycemic control is essential for preventing perioperative inflammatory response and improving patient outcome. Furthermore, recent studies have shown that reducing the variability of blood glucose concentration may play an important role in glucose management (9). 
However, it has also been shown that intensive insulin treatment is sometimes difficult to perform when using sliding-scale manual insulin injection because hypoglycemia occurs frequently in spite of frequent blood glucose testing. It has therefore been suggested that continuous blood glucose monitoring would be beneficial for maintaining target blood glucose levels.

A recent report advocated the usefulness of a closed-loop system (artificial endocrine pancreas), which provides continuous monitoring and strict control of blood glucose. The STG-22 (Nikkiso, Tokyo, Japan) is a novel artificial endocrine pancreas with a closed-loop glycemic control system that provides continuous blood glucose monitoring by means of a glucose sensor electrode and subsequent automatic insulin and glucose infusion to maintain appropriate blood glucose levels (10). Our group also reported the usefulness of a closed-loop system providing continuous monitoring and strict control of peri-operative blood glucose in patients during surgery (11). An artificial endocrine pancreas may thus be useful for such accurate and continuous blood glucose monitoring and strict glycemic control during liver transplantation.

The purpose of this study was to evaluate the usefulness of the STG-22 closed-loop system for the continuous monitoring and control of intraoperative blood glucose of patients undergoing living-donor liver transplantation. Our final goal is the establishment of a new superior perioperative blood glucose control method using an artificial endocrine pancreas during liver transplantation.

\section{PATIENTS AND METHODS}

This investigation conformed to the principles outlined in the Declaration of Helsinki. The study protocol was approved by the Ethics Committee on $\mathrm{Hu}-$ man Studies of Tokushima University Hospital, and written informed consent was obtained from each patient. Sixteen patients undergoing living-donor liver transplantation were enrolled in this study.

\section{Intraoperative Management}

For this comparative utility assessment study, cannulae were inserted into a peripheral vein and a radial artery in the operating theater. Standard monitoring included pulse oximetry, lead II of the electrocardiogram for heart rate and automated STsegment trend analysis, and end-tidal capnography.
General anesthesia was induced using intravenous fentanyl and propofol. Neuromuscular blocking was obtained by rocuronium. Following tracheal intubation, the lungs were mechanically ventilated with a combination of oxygen and air and the tidal volume was adjusted to produce normocapnia (end-tidal carbon dioxide between 30 and $40 \mathrm{mmHg}$ ). Anesthesia was maintained with isoflurane and fentanyl. After induction of general anesthesia and tracheal intubation, a pulmonary artery catheter and a central venous catheter were inserted through the right internal jugular vein.

Glucose levels were controlled with either a manual injection of insulin based on a commonly used sliding scale (manual insulin group, $\mathrm{n}=8$ ) or a programmed infusion of insulin determined by the control algorithm of the STG-22 (programmed insulin group, $n=8$ ). Regular insulin human (Humulin $\mathrm{R}$; Eli Lilly and Company, Indianapolis, USA) was used for both groups. Rapidlab860 (Bayer Medical, Tokyo, Japan) was used for conventional blood glucose assessment. For the programmed insulin group, a 20G intravenous catheter (Insyte ; Becton Dickinson Infusion Therapy Systems, Sandy, UT) was inserted after anesthesia induction into a peripheral forearm vein and connected to the STG-22 for continuous blood glucose monitoring. The target glucose level of $80-150 \mathrm{mg} / \mathrm{d} \ell$ was attained for both groups as determined with a radial arterial catheter used for intermittent blood glucose sampling. Samples were obtained during surgery after an established protocol of discarding $2 \mathrm{ml}$ of blood before withdrawal of the actual blood sample by the same anesthesiologist.

\section{Equipment}

The STG-22 provides continuous blood glucose monitoring through a dual lumen catheter blood sampling technique, a high-quality roller pump (multichannel pump) and a glucose sensor electrode with a glucose oxidase membrane. Before starting blood glucose monitoring, a two-point calibration was performed using a standard solution for internal calibration (glucose concentration : $0 \mathrm{mg} / \mathrm{d} \ell$ ) and a standard glucose solution $(200 \mathrm{mg} / \mathrm{d} \ell)$. During blood glucose monitoring, internal calibration using the standard solution for internal calibration was performed automatically every $4 \mathrm{~h}$. After calibration of the equipment, blood was sampled continuously from the peripheral vein at a rate of $2 \mathrm{ml} / \mathrm{h}$ and continuously diluted with a heparinized isotonic solution. The diluted blood was further diluted with an 
isotonic buffer solution of phosphoric acid, $\mathrm{pH}$ 7.4, after which the glucose sensor electrode was exposed to the sampled blood. The multichannel pump and the glucose sensor electrode both had an accuracy of $\pm 5 \%$. The accuracy and reliability of this system during and after surgery have been confirmed $(12,13)$.

\section{Data collection}

The resultant 78 scores for paired blood glucose levels obtained with continuous glucose measurements (STG-22) and with conventional intermittent glucose measurements (Rapidlab 860) were compared. For each patient, the mean and standard deviation (SD) of blood glucose concentration during surgery (Glu-Ave and Glu-SD) were calculated as the arithmetical mean and SD of the entire set of measurements. To evaluate relative variability, the coefficient of variability (Glu-CV $=$ Glu-SD $\times 100$ / Glu-Ave) (9) was also calculated and the maximum blood glucose level of each patient (Glu-Max) was adopted as the highest value occurring during surgery.

\section{Statistical analysis}

The accuracy of continuous glucose measurements (STG-22) and of conventional intermittent glucose measurements (Rapidlab 860) during liver transplantation was compared by means of the Bland-Altman plot (14). In addition, the values obtained with the two methods were subjected to correlation analysis. Other data were analyzed with the Mann-Whitney U-test or Fisher's exact probability test. Values are expressed as mean $\pm \mathrm{SD}$, and $\mathrm{P}<$ 0.05 was considered to be statistically significant.

\section{RESULTS}

Figure 1 (left) shows a close correlation $(r=0.88)$ between continuous glucose measurements (STG22) and conventional intermittent glucose measurements (Rapidlab 860) for the programmed insulin group. Significant agreement was also observed when the differences between the two measurements were plotted against their mean value (Figure 1, right).

Patient characteristics presented in Table 1 show no significant differences between the two groups for any of the values listed. Figure 2 shows a typical example of intraoperative blood glucose changes in the manual insulin group. The blood glucose concentration increased gradually during the preanhepatic phase of transplantation, and significantly so during reperfusion of the donor liver. Intraoperative blood glucose levels of the manual insulin group fluctuated very widely (minimum : $85 \mathrm{mg} / \mathrm{d} \ell$, maximum : $356 \mathrm{mg} / \mathrm{d} \ell$ ), so that strict control was often difficult. On the other hand, stable blood glucose values were maintained automatically (minimum : $82 \mathrm{mg} / \mathrm{d} \ell$ : maximum : $205 \mathrm{mg} / \mathrm{d} \ell$ ) for the programmed insulin group. Figure 3 shows a typical example from the programmed insulin group. Continuous monitoring and control of blood glucose were successfully attained with the aid of the artificial endocrine pancreas. Before clamping of the hepatic artery and portal vein, the blood glucose level tended to decrease, but hypoglycemia was avoided by means of appropriate glucose administration. The blood glucose level suddenly started to increase after reperfusion of the portal vein and hepatic artery, but only to a maximum of $150 \mathrm{mg} / \mathrm{d} \ell$, and the level
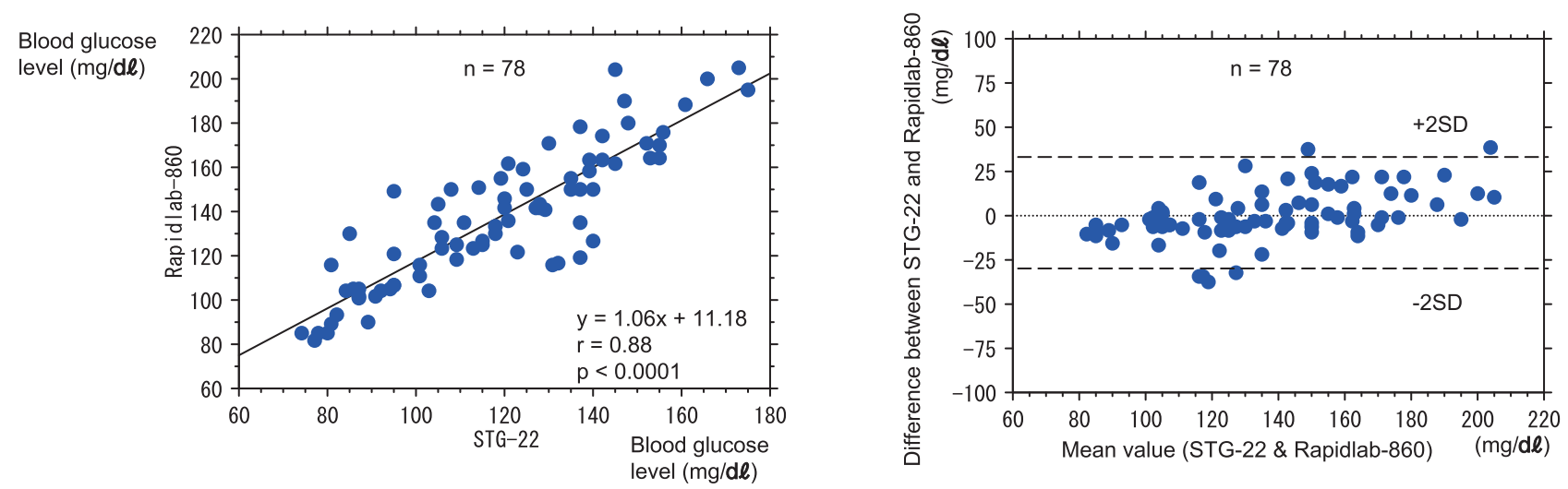

Figure 1. Accuracy of continuous blood glucose monitoring during liver transplantation

Left : Correlations between continuous glucose measurements (STG-22) and conventional intermittent glucose measurements (Rapidlab 860)

Right: Differences between the two measurements plotted against their mean (Bland-Altman plot of continuous glucose measurements (STG-22) and conventional intermittent glucose measurements (Rapidlab 860)) 
Table 1: $\quad$ Patient characteristics

\begin{tabular}{|c|c|c|c|}
\hline & $\begin{array}{l}\text { Manual insulin group } \\
(\mathrm{n}=8)\end{array}$ & $\begin{array}{l}\text { Programmed insulin group } \\
(\mathrm{n}=8)\end{array}$ & $\mathrm{P}$-value \\
\hline Age (years) & $56.0 \pm 3.7$ & $48.6 \pm 12.4$ & NS \\
\hline Height $(\mathrm{cm})$ & $158.0 \pm 8.5$ & $159.4 \pm 8.9$ & NS \\
\hline Weight $(\mathrm{kg})$ & $59.2 \pm 6.5$ & $59.1 \pm 14.5$ & NS \\
\hline Gender (male/female) & $3 / 5$ & $1 / 7$ & NS \\
\hline Previous diabetes mellitus (Yes/No) & $2 / 6$ & $1 / 7$ & NS \\
\hline Fasting blood glucose $(\mathrm{mg} / \mathrm{d} \ell)$ & $104.1 \pm 21.4$ & $91.4 \pm 10.9$ & NS \\
\hline HbA1c (\%) & $5.5 \pm 2.1$ & $4.2 \pm 0.7$ & NS \\
\hline \multicolumn{4}{|l|}{ Underlying liver disease } \\
\hline Hepatitis B & 5 & 3 & \\
\hline Hepatitis C & 3 & 3 & \\
\hline Liver cirrhosis & 7 & 6 & \\
\hline Hepatocellular carcinoma & 3 & 4 & \\
\hline Estimated blood loss $(\mathrm{m} \ell)$ & $3617 \pm 3498$ & $2551 \pm 3199$ & NS \\
\hline Operation time (min) & $780 \pm 113$ & $772 \pm 118$ & NS \\
\hline Anesthesia time (min) & $918 \pm 105$ & $907 \pm 122$ & NS \\
\hline
\end{tabular}

NS : not significant, Mean $\pm \mathrm{SD}$

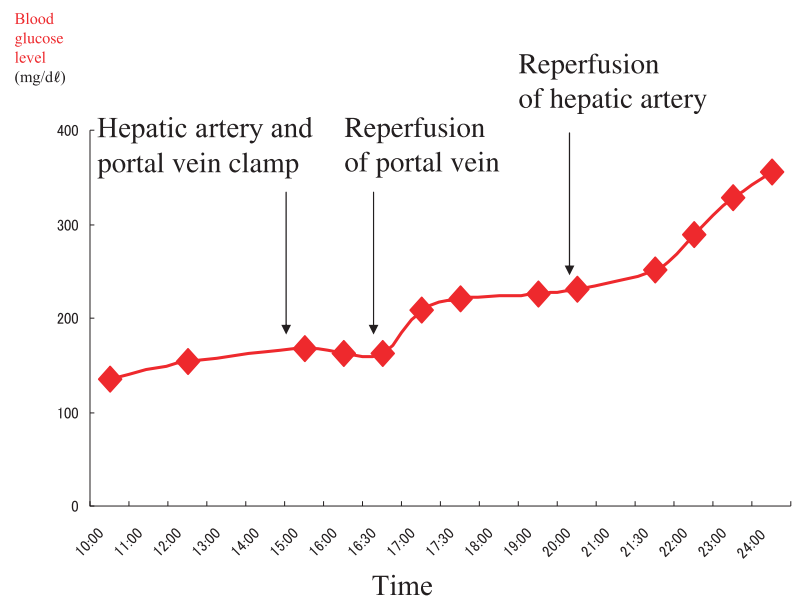

Figure 2. Continuous blood glucose monitoring during liver transplantation (typical example from the manual insulin group)

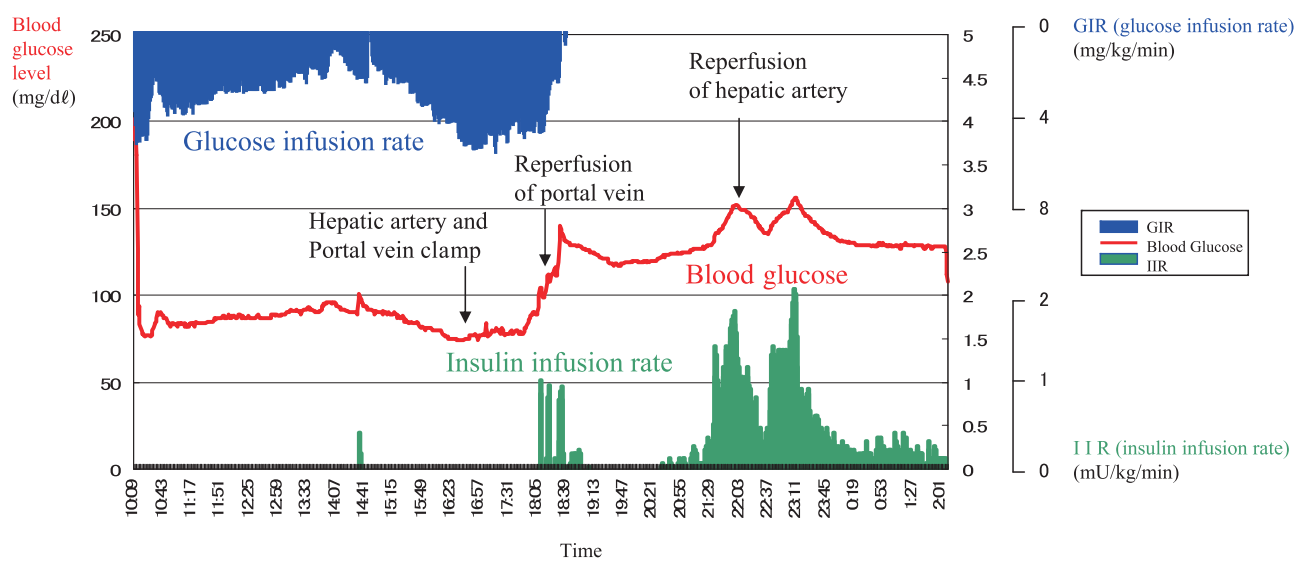

Figure 3. Intensive insulin therapy during liver transplantation using the artificial endocrine pancreas (typical example from the programmed insulin group)

GIR : glucose infusion rate; IIR : insulin infusion rate

During the preanhepatic phase, hypoglycemia was avoided by means of continuous glucose infusion, and after reperfusion, hyperglycemia was avoided by means of continuous insulin infusion. 
was controlled by means of appropriate insulin administration.

Figure 4 shows the results of blood glucose management. Glu-Ave, Glu-SD, and Glu-Max for the programmed insulin group $(135.1 \pm 15.3,27.3 \pm$ $7.7,179.1 \pm 23.7 \mathrm{mg} / \mathrm{d} \ell$, respectively) were lower than for the manual insulin group $(188.1 \pm 33.2$, $50.8 \pm 16.6,272.8 \pm 64.4 \mathrm{mg} / \mathrm{d} \ell$ ), while Glu-CV for the programmed insulin group was also lower than that for the manual insulin group $(20.1 \pm 4.9 \%$ vs. $26.9 \pm 6.1 \%)$. The overall success rate during surgery, expressed as a percentage of all blood glucose measurements within the target level range, was $77.5 \pm 20.6 \%$ for the programmed insulin group and $42.0 \pm 8.8 \%$ for the manual insulin group $(\mathrm{P}<0.05)$ (Figure 5, left). The total amount of insulin administered per patient during surgery was not significantly different for the two groups $(28.2 \pm 14.9 \mathrm{IU}$ vs. $20.2 \pm 20.8 \mathrm{IU}$ ) (Figure 5, right). No hypoglycemia was detected in either group.
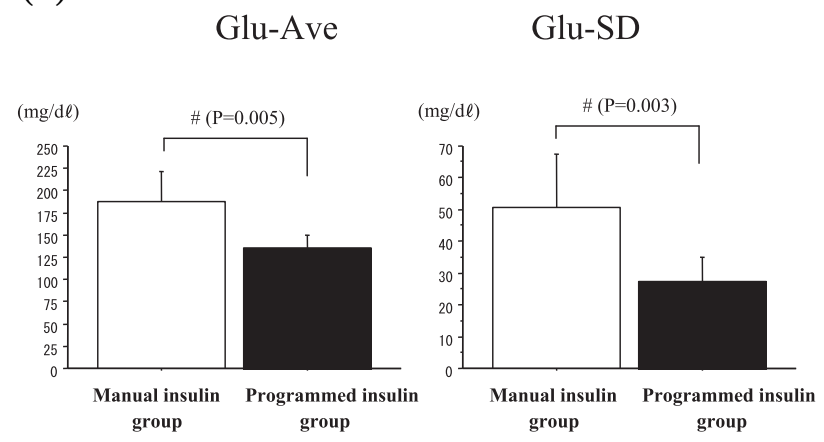

(B)
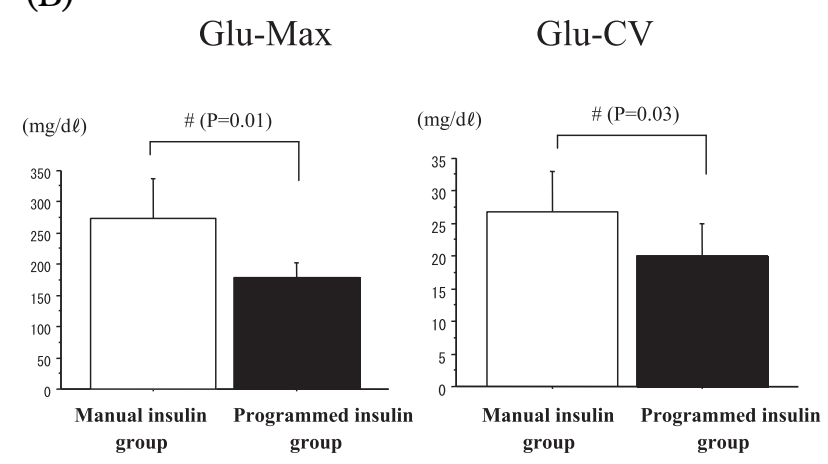

Figure 4. Glucose management

A. Comparison of Glu-Ave and Glu-SD for the two groups B. Comparison of Glu-Max and Glu-CV for the two groups Glu-AVE : the mean of blood glucose concentration during surgery ; Glu-SD : the standard deviation (SD) of blood glucose concentration during surgery ; Glu-Max : the maximum blood glucose level in each patient; Glu-CV : the coefficient of variability (Glu-CV = Glu-SD $\times 100 /$ Glu-Ave)

\# : Significant $(\mathrm{P}<0.05)$ difference between groups. Values are expressed as mean $\pm \mathrm{SD}$.

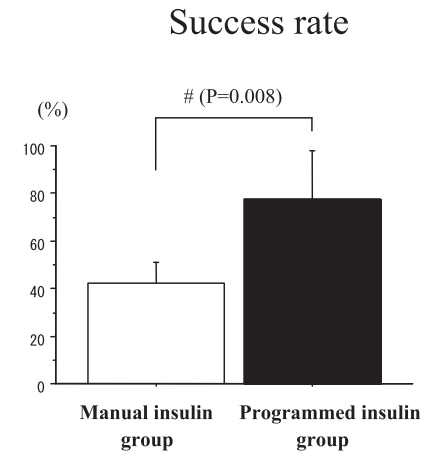

Total amount of insulin

Figure 5. Comparison of success rate and total amount of insulin

NS : not significant. Values are expressed as mean \pm SD.

\section{DISCUSSION}

This is the first report of a closed-loop glucosesensing and insulin delivery system, in this case the STG-22, being used for intraoperative management during liver transplantation. Our results showed that the programmed insulin group showed a higher success rate for attaining the target blood glucose level than the manual insulin group, even though the total amount of insulin administered per patient during surgery was not significantly different for the two groups. No hypoglycemia was detected in either group. In addition, stable blood glucose management could be achieved with much less blood glucose variability in the programmed than in the manual insulin group.

During liver transplantation, blood glucose levels change dramatically (3). As the liver plays an important role in the maintenance of the plasma glucose, liver transplantation causes disorders in glucose metabolism. It is easy for hypoglycemia to occur when insufficient glycogen is stored in the damaged liver during the preanhepatic phase. In anticipation of such a heightened tendency for hypoglycemia to occur, glucose consumption continues while the glucose supply stops from the liver during the anhepatic phase. On the other hand, plasma glucose suddenly increases after reperfusion of the donor liver as a result of release of the glucose load from the donor liver or due to blood transfusion. Of special note is that some studies have found that intraoperative hyperglycemia during liver transplantationation was associated with an increased risk of postoperative infection and mortality $(5,6)$. Hyperglycemia is a direct cause of toxicity, and oxidative stress in the cell due to high mitochondria peroxide production may increase (15). The clinical importance of hyperglycemia seems to be that morbidity and 
mortality increase as was observed in a group of critically ill patients. Because insulin performs a vital function not only in blood glucose control, but also as an anti-inflammatory and antioxidant agent, the strict maintenance of normoglycemia by means of intensive insulin treatment seems to reduce mortality as well as functional disorders of the liver kidney, and the endothelium of the critically ill patient. (16). Because insulin inhibits reactive oxygen species production by controlling the proinflamatory effects of $\mathrm{NF} \kappa \mathrm{B}$, activator protein 1 (AP-1), early growth response 1 (Egr-1) and high-mobility group box 1 (HMGB1) (16-18). Strict intraoperative glycemic control, possibly using insulin infusions, may thus improve outcomes following liver transplantation.

Intensive insulin therapy is used for critically ill patients, and its specific aims are multiple organ protection and the prevention and treatment of infection by normalizing and maintaining blood glucose levels normal levels $(7,8)$. Van den Berghe et al. (7) were the first to report in 2001 that tight glycemic control (maintaining blood glucose levels at 80-110 $\mathrm{mg} / \mathrm{d} \ell$ ) improved morbidity and mortality in the surgical intensive care unit (ICU). They demonstrated that the overall ICU mortality rates dropped from $8 \%$ to $4.6 \%$ and from $20.2 \%$ to $10.6 \%$ for patients requiring more than 5 days of intensive care. Many modified versions of the original therapy have since been introduced with the aim of attaining a blood glucose level upper limit of around 140 to $180 \mathrm{mg} / \mathrm{d} \ell$ (19-21). The most notable reduction in mortality resulting from this therapy involves death due to multiple-organ failure with a proven septic focus. Lower blood glucose levels have been recommended in international consensus guidelines as a means of improving patient outcomes. The results of these trials indicate that intraoperative blood glucose control that maintains blood glucose levels $\leqq 150 \mathrm{mg} / \mathrm{d} \ell$ should be considered of vital importance.

Some recent negative reports about intensive insulin therapy have led to a reconsideration of such therapy (22-24). The results of the NICE-SUGAR study were presented in the March issue of the New England Journal of Medicine in 2009 (22). Contrary to expectations, the mortality for the intensive therapy group (target blood sugar value : $81-108$ $\mathrm{mg} / \mathrm{d} \ell$ ) after 90 days was higher (27.5\% vs. $24.9 \%)$ than that for the conventional therapy group (target blood sugar value : $144-180 \mathrm{mg} / \mathrm{d} \ell$ ). Do these findings mean that intensive insulin therapy should be discarded entirely? Although this is probably not the case, it is clear that there is an urgent need for the establishment of a new blood glucose control method that does not cause hypoglycemia. In addition, a recent study has shown that reducing variability of blood glucose concentration may play an important role in glucose management (9).

In December 2007, our clinical team at Tokushima University initiated a clinical trial to evaluate the efficacy of the artificial pancreas for strict perioperative glycemic control of intra-operative patients. Two STG-22 artificial pancreas systems were put into clinical use at our institution for intra- and postoperative glucose control (11). An artificial pancreas (STG-22) was developed by Nikkiso (Tokyo, Japan), which collects venous blood continuously for continuous blood glucose monitoring. Furthermore, this system carries out direct measurements of blood glucose and can therefore detect and show rapid changes in blood glucose levels. The reliability and accuracy of continuous blood glucose monitoring with the STG-22 has been verified in several studies, such as the ones by Yamashita and colleagues $(12,13)$. In our study, blood glucose measured continuously during liver transplantation with the STG-22 correlated strongly with measurements obtained intermittently with a conventional laboratory glucometer.

Previous studies reported that no surgical site infection occurred after liver resection in a group whose perioperative blood glucose was controlled with an artificial pancreas $(25,26)$, indicating that lack of perioperative blood glucose control is related to the incidence of postoperative infectious complications and longer hospitalization. As already mentioned, the artificial pancreas is a safe and effective device for strict perioperative glycemic control without hypoglycemia for patients who undergo hepatic resection for liver diseases. In addition, the frequent blood sugar measurement contributes to a reduction in work load for the staff and the shorter hospitalization to a reduction in incident, medical expenses (27). The next-generation artificial endocrine pancreas, equipped with a disposable and modular tubing circuit with an auto-priming function, automatic calibration with quick response in sensor setup and a compact structure, is also available (28). However, establishment of a new perioperative blood glucose control method using an artificial endocrine pancreas to make effective use of this advanced equipment is urgently needed.

In conclusion, strict perioperative glycemic control is effective for the protection of many organs and a reduced incidence of infection so that it is 
reasonable to conclude that it can lead to improved prognosis for liver transplantation. However, such control is difficult to implement due to the need for intermittent blood glucose measurements and the manual administration of insulin. The STG-22 closed-loop system (an artificial endocrine pancreas) is effective for maintaining strict blood glucose control during living-donor liver transplantation with minimal variability in blood glucose concentration. However, our clinical study has certain limitations : 1) the sample size was small ; 2) it was not a randomized control study ; 3 ) no outcome study was performed. Determination of the optimal target blood glucose range during liver transplantation and the long-term clinical implications of use of the artificial pancreas for perioperative glycemic control are important questions for further investigations of larger study groups.

\section{FINANCIAL SUPPORT}

This study was supported by intramural departmental funds

\section{CONFLICT OF INTERESTS}

None

\section{REFERENCES}

1. Shimada M, Fujii M, Morine $Y$, Imura $S$, Ikemoto $\mathrm{T}$, Ishibashi $\mathrm{H}$ : Living-donor liver transplantation : present status and future perspective. J Med Invest $52: 22-32,2005$

2. Carton EG, Plevak DJ, Kranner PW, Rettke SR, Geiger HJ, Coursin DB : Perioperative care of the liver transplant patient: Part 2. Anesth Analg 78 : 382-399, 1994

3. Atchison SR, Rettke SR, Fromme GA, Janossy TA, Kunkel SE, Williamson KR, Perkins JD, Rakela J : Plasma glucose concentrations during liver transplantation. Mayo Clin Proc 64 : 241-245, 1989

4. Wallia A, Parikh ND, Molitch ME, Mahler E, Tian L, Huang JJ, Levitsky J : Posttransplant hyperglycemia is associated with increased risk of liver allograft rejection. Transplantation 89 : 222-226, 2010

5. Ammori JB, Sigakis M, Englesbe MJ, O'Reilly
M, Pelletier SJ : Effect of intraoperative hyperglycemia during liver transplantation. J Surg Res 140 : 227-233, 2007

6. Park C, Hsu C, Neelakanta G, Nourmand H, Braunfeld M, Wray C, Steadman RH, Hu KQ, Cheng RT, Xia VW : Severe intraoperative hyperglycemia is independently associated with surgical site infection after liver transplantation. Transplantation 87 : 1031-1036, 2009

7. Van den Berghe G, Wouters P, Weekers F, Verwaest C, Bruyninckx F, Schetz M, Vlasselaers D, Ferdinande P, Lauwers P, Bouillon R: Intensive insulin therapy in the critically ill patients. N Eng J Med 345 : 13591367, 2001

8. Van den Berghe G, Wouters PJ, Bouillon R, Weekers F, Verwaest C, Schetz M, Vlasselaers D, Ferdinande P, Lauwers P : Outcome benefit of intensive insulin therapy in the critically ill : insulin dose versus glycemic control. Crit Care Med 31 : 359-366, 2003

9. Egi M, Bellomo R, Stachowski E, French CJ, Hart G : Variability of blood glucose concentration and short-term mortality in critically ill patients. Anesthesiology 105 : 244-252, 2006

10. Hanazaki K, Nosé Y, Brunicardi FC : Artificial endocrine pancreas. J Am Coll Surg 193 : 310322,2001

11. Kawahito S, Kitahata H, Kitagawa T, Oshita S : Intensive insulin therapy during cardiovascular surgery. J Med Invest 57 : 191-204, 2010

12. Yamashita $\mathrm{K}$, Okabayashi $\mathrm{T}$, Yokoyama T, Yatabe T, Maeda H, Manabe M, Hanazaki K : The accuracy of a continuous blood glucose monitor during surgery. Anesth Analg 106; 160-163 : 2008

13. Yamashita K, Okabayashi T, Yokoyama T, Yatabe T, Maeda H, Manabe M, Hanazaki K: Accuracy and reliability of continuous blood glucose monitor in post-surgical patients. Acta Anaesthsiol Scand 53 : 66-71, 2009

14. Bland JM, Altman DG : Statistical methods for assessing agreement between two methods of clinical measurement. Lancet 8 : 307-310, 1986

15. Kawahito S, Kitahata H, Oshita S : Problems associated with glucose toxicity : role of hyperglycemia-induced oxidative stress. World J Gastroenterol 15 : 4137-4142, 2009

16. Dandona P, Aljada A, Mohanty P, Ghanim H, Hamouda W, Assian E, Ahmad S : Insulin inhibits intranuclear nuclear factor $\mathrm{\kappa B}$ and stimulates $i \kappa B$ in mononuclear cells in obese subjects : 
evidence for an anti-inflammatory effect? J Clin Endocrinol Metab 86 : 3257-3265, 2001

17. Aljada A, Ghanim H, Mohanty P, Kapur N, Dandona $\mathrm{P}$ : Insulin inhibits the pro-inflammatory transcription factor early growth response gene-1 (Egr)-1 expression in mononuclear cells (MNC) and reduces plasma tissue factor (TF) and plasminogen activator inhibitor-1 (PAI-1) concentrations. J Clin Endocrinol Metab 87 : 1419-1422, 2002

18. Aljada A, Ghanim H, Mohanty P, Syed T, Bandyopadhyay A, Dandona P : Glucose intake induces an increase in activator protein 1 and early growth response 1 binding activities, in the expression of tissue factor and matrix metalloproteinase in mononuclear cells, and in plasma tissue factor and matrix metalloproteinase concentrations. Am J Clin Nutr 80 : 5157, 2004

19. Finney SJ, Zekveld C, Elia A, Evans TW : Glucose control and mortality in critically ill patients. JAMA 290 : 2041-2047, 2003

20. Krinslery JS : Effect of an glucose management protocol on the mortality of critically ill adult patients. Mayo Clin Proc 79 : 992-1000, 2004

21. Lipshultz AKM, Gropper MA : Perioperative glycemic control : an evidence-based review. Anesthesiology 110 : 408-421, 2009

22. The NICE-SUGAR study Investigators : Intensive versus conventional glucose control in critically ill adults. N Eng J Med 360 : 12831297, 2009

23. Wiener RS, Wiener DC, Larson RJ : Benefits and risks of tight glucose control in critically ill adults : a meta-analysis. JAMA 300 : 933-944, 2008

24. Arabi YM, Dabbagh OC, Tamim HM, AlShimemeri AA, Memish ZA, Haddad SH, Syed SJ, Giridhar HR, Rishu AH, Al-Daker MO, Kahoul SH, Britts RJ, Sakkijha MH : Intensive versus conventional insulin therapy : a randomized controlled trial in medical and surgical critically ill patients. Crit Care Med 36 : 3190-3197, 2008

25. Okabayashi T, Nishimori I, Yamashita K, Sugimoto T, Yatabe T, Maeda H, Kobayashi M, Hanazaki K : Risk factors and predictors for surgical site infection after hepatic resection. J Hosp Infect 73 : 47-53, 2009

26. Hanazaki K, Maeda H, Okabayashi T : Relationship between perioperative glycemic control and postoperative infections. World J Gastroenterol 15 : 4122-4125, 2009

27. Hanazaki K, Maeda H, Okabayashi T : Tight perioperative glycemic control using an artificial endocrine pancreas. Surg Today $40: 1-7,2010$

28. Tsukamoto Y, Okabayashi T, Hanazaki K. Progressive artificial endocrine pancreas : the era of novel perioperative blood glucose control for surgery. Surg Today $41:$ 1344-1351, 2011 